\title{
Reducing factor XI with antisense oligonucleotides superior to endoxaparin for postoperative venous thromboembolism
}

Antithrombotic therapies are often associated with an increased risk of bleeding, but factor XI has been identified as a potential target for inhibiting thrombus formation without interfering with haemostasis. In a report published in the New England Journal of Medicine, antisense oligonucleotides that reduce expression of factor XI decreased the incidence of postoperative venous thromboembolism after knee arthroplasty.

In this open-labelled study, 300 patients undergoing primary unilateral total knee arthroplasty were randomly allocated to receive either $200 \mathrm{mg}$ or $300 \mathrm{mg}$ of FXI-ASO (a second-generation antisense oligonucleotide that specifically reduces
mRNA levels of factor XI) starting 36 days before surgery, or $40 \mathrm{mg}$ of the anticoagulant enoxaparin starting the evening before or 6-8 $\mathrm{h}$ after surgery. The primary efficacy outcome (incidence of thromboembolism detected by venography 8-12 days after surgery) occurred in $27 \%(36 / 134)$ of patients who received $200 \mathrm{mg}$ FXI-ASO, $4 \%$ (3/71) of patients who received $300 \mathrm{mg}$ FXI-ASO, and 30\% (21/69) of patients who received enoxaparin, indicating that treatment with $300 \mathrm{mg}$ FXI-ASO was superior to enoxaparin $(P<0.001)$. The rate of bleeding was numerically, but not significantly, lower with 300 mg FXI-ASO than with enoxaparin (3\% versus $8 \%$ ).
In an accompanying editorial, Robert Flaumenhaft, who was not involved in the investigation, cautions that "a larger study will be required to evaluate whether targeting factor XI spares haemostasis". Nevertheless, these results suggest that strategies to lower factor XI levels can be more effective at reducing postoperative thrombosis than conventional anticoagulants, and without increasing the risk of bleeding.

João H. Duarte

Original articles Büller, H. R. et al. Factor XI antisense oligonucleotide for prevention of venous thrombosis. N. Engl. J. Med. doi:10.1056/NEJMoa1405760 | Flaumenhaft, R. Making (anti)sense of factor XI in thrombosis. N. Eng. J. Med. doi:10.1056/NEJMe1413874 